\title{
Efficacy of Ultrasonography Guided Stellate Ganglion Blockade in the Stroke Patients with Complex Regional Pain Syndrome
}

\author{
Seung Don Yoo, M.D., Sang Soo Jung, M.D., Hee-Sang Kim, M.D., Dong Hwan Yun, M.D., \\ Dong Hwan Kim, M.D., Jinmann Chon, M.D., Dong Whan Hong, M.D.
}

Department of Physical Medicine and Rehabilitation, Kyung Hee University College Medicine, Seoul 130-702, Korea

\begin{abstract}
Objective To compare the efficacy of ultrasonography guided stellate ganglion block (US-SGB) with that of blind SGB in management of the stroke patients with complex regional pain syndrome (CRPS) type 1.

Method Forty-two patients with post-stroke CRPS were randomly assigned to either US-guided SGB (22 patients) or blind SGB group (20 patients). The mean age of US-guided SGB and blind SGB groups was $61.3 \pm 5.6$ years and $59.1 \pm 4.5$ years. We performed two blockades at 7 -day intervals on the affected side of patients with CRPS. Pain intensity, using a visual analog score (VAS), score of CRPS clinical severity, and the amounts of affected hand swelling with a hand volumeter were assessed before, 2 weeks and 4 weeks after treatment.

Results In both groups, VAS and the amount of hand swelling were significantly decreased after 2 weeks and after 4 weeks. Between two groups, VAS difference of US-guided SGB group and that of blind SGB group were 2.61 \pm 1.09 , $1.88 \pm 0.62$ at 2 weeks and $3.67 \pm 1.03,3.13 \pm 0.62$ at 4 weeks, respectively. US-guided SGB group showed more significant improvement in mean change of VAS compared to the blind SGB group ( $\mathrm{p}$-value $<0.05)$.

Conclusion Both US-guided SGB and blind SGB techniques were effective in relieving pain in subacute stroke patients with CRPS. US-guided SGB was better in pain relief but has no advantages in reduction of hand swelling in this study.
\end{abstract}

Key Words Complex regional pain syndrome, Stroke, Ultrasonography guided Stellate ganglion blockade

Received May 22, 2012; Accepted July 18, 2012

Corresponding author: Sang Soo Jung

Department of Physical Medicine and Rehabilitation, Kyung Hee University College Medicine, 149 Sangil-dong, Gangdong-gu, Seoul 134727 , Korea

Tel: +82-2-440-6171, Fax: +82-2-440-6434, E-mail: sangsoo-haha@ hanmail.net

(c) This is an open-access article distributed under the terms of the Creative Commons Attribution Non-Commercial License (http:// creativecommons.org/licenses/by-nc/3.0) which permits unrestricted noncommercial use, distribution, and reproduction in any medium, provided the original work is properly cited.

Copyright $\odot 2012$ by Korean Academy of Rehabilitation Medicine

\section{INTRODUCTION}

Complex regional pain syndrome (CRPS), the current diagnostic label for the syndrome historically referred to as reflex sympathetic dystrophy, causalgia, and a variety of other terms, can be difficult to treat. ${ }^{1}$ CRPS manifests as persistent burning pain on a limb, with a region of intense allodynia, hyperalgesia, and objective evidence of local autonomic dysfunction and trophic changes. ${ }^{2}$ Dysfunction of the sympathetic nervous system is believed to be attributed to changes in microcirculatory disturbances 
resulting from extensive sprouting of noradrenergic sympathetic fibers in the sensory ganglia and the peripheral nerves. $^{2-5}$

Stroke is a common cause of CRPS, and between $1.5 \%$ and $61 \%$ of patients following stroke have been reported to develop CRPS.$^{6-8}$ Various management modes for CRPS have been introduced but only a few techniques have been effective. The sympathetic nervous system has been implicated in the pathophysiology of CRPS, and consequently, sympathetic nerve blockade (SGB) is widely used for the treatment of CRPS. ${ }^{9}$

Classical "blind" injection of anesthetic at the C6 level has a long history, but its reliability in achieving blockade of the sympathetic trunk and stellate ganglion remains uncertain. In addition, blind injection is associated with a variety of adverse effects and complications, such as intravascular injection, formation of hematomas and temporary paralysis of the recurrent laryngeal nerve..$^{10-13}$

A method of stellate ganglion blockade under ultrasonography (US) guidance was described in 1995, but only recently gained popularity. Kapral et al. ${ }^{12}$ found that compared with the blind technique, ultrasonographyguided SGB used a lower volume of local anesthetics (5 $\mathrm{ml}$ rather than $8 \mathrm{ml}$ ) and there were more rapid onsets of Horner's syndrome. Application of US for sympathetic nerve blockade has proven to be useful for the swift and accurate placement of local anesthetics on a targeted area. Thus, it might reduce the required volume of local anesthetics compared with blind technique.

The purpose of this prospective study was to evaluate the effectiveness of US-guide SGB by comparing with the blind SGB in post-stroke CRPS patients in reducing pain and swelling of the affected limb.

\section{MATERIALS AND METHODS}

Among 59 patients with the presence of CRPS following a cerebrovascular accident (CVA), patients whose CVA occurred in 2009 or 2010 were selected.

The inclusion criteria were the presence of regional pain and sensory changes of hemiplegic unilateral upper extremity following a stroke; pain was associated with findings such as abnormal skin color, temperature change, abnormal sudomotor activity or edema; no distribution of the pain of a single nerve in the extremity. Seventeen subjects were excluded from this study if they had a history of upper extremity cellulitis, other autonomic nerve disease, peripheral nerve disease, bleeding disorder or severe cognitive impairment.

We therefore studied 42 patients with CRPS following stroke [19 right (45.3\%) and 23 left (54.7\%)] and patient signed informed consent. All 42 patients had the modified diagnostic criteria for CRPS as International Association for the Study of Pain (IASP) ${ }^{14}$ Twenty-five patients were women (59.6\%) and 17 (40.4\%) were men. All patients were divided randomly into US-guided SGB group (22 patients) and blind SGB group (20 patients). The mean age was $61.3 \pm 5.6$ (range $34-77$ ) years and $59.1 \pm 4.5$ (range 36-71) years and the mean duration since stroke occurred were $2.8 \pm 1.1$ (range 1-7) months, $2.3 \pm 0.9$ (range 1-6) months, respectively (Table 1).

Kapral et al. ${ }^{12}$ reported that US-guided SGB, by comparing with the blind technique, used a lower volume of local anesthetics and proved to be more effective. In this study, the blind SGB group patients and US-guided SGB group patients received the procedure using a volume of $10 \mathrm{ml}$ of $0.5 \%$ lidocaine or $5 \mathrm{ml}$ of $0.5 \%$ lidocaine, respectively. SGB was performed two times with an interval of 1

Table 1. Clinical Characteristics of Subjects

\begin{tabular}{lcc}
\hline & Blind group $(\mathbf{n}=\mathbf{2 0})$ & US-guided group $(\mathbf{n = 2 2})$ \\
\hline Male/female (n) & $11 / 9$ & $14 / 10$ \\
Age (mean age \pm SD) & $59.1 \pm 4.5$ & $61.3 \pm 5.6$ \\
Ischemic/hemorrhage & $12 / 8$ & $13 / 9$ \\
Stroke duration (mean \pm SD, months) & $2.3 \pm 0.9$ & $2.8 \pm 1.1$ \\
CRPS stage (n) & & \\
1 & $18(90 \%)$ & $21(87.5 \%)$ \\
2 & $2(10 \%)$ & $3(12.5 \%)$ \\
$3-4$ & 0 & 0 \\
\hline
\end{tabular}

SD: Standard deviation, CRPS: Complex regional pain syndrome 
week between each treatments. ${ }^{12}$

The anterior paratracheal approach was used in the procedure on the cervical sympathetic chain without fluoroscopic guidance in blind group. A 22 -gauge, $5-\mathrm{cm}$ needle was perpendicularly inserted to the skin until bone contact was made and then withdrawn to rest anteriorly to the precervical fascia. The patient was encouraged to lie in the supine position for 3 minute after the injection and then developed injected-sided Horner's syndrome. ${ }^{15}$

In US-guided group, procedure was performed using same needle at same posture. After passing through the skin and subcutaneous tissue and stabilizing the needle, a 3-12 MHz linear array probe of HD11-XE ${ }^{\circledR}$ (Philips, Washington, USA) was used to verify the position of the needle. The needle was shown to be aiming towards the thyroid tissue anteriorly and then the esophagus posteriorly. At this point the needle was withdrawn, reinserted obliquely, and advanced with real-time US so that the needle tip lay anterior to the longus coli muscle (anterior to $\mathrm{C} 6$ transverse process) (Fig. 1). After negative aspiration, $5 \mathrm{ml}$ of lidocaine $0.5 \%$ were injected.

The primary outcome was evaluated by pain intensity and the amount of hand swelling. Pain intensity was evaluated by using a $10 \mathrm{~cm}$ visual analog scale (VAS) in

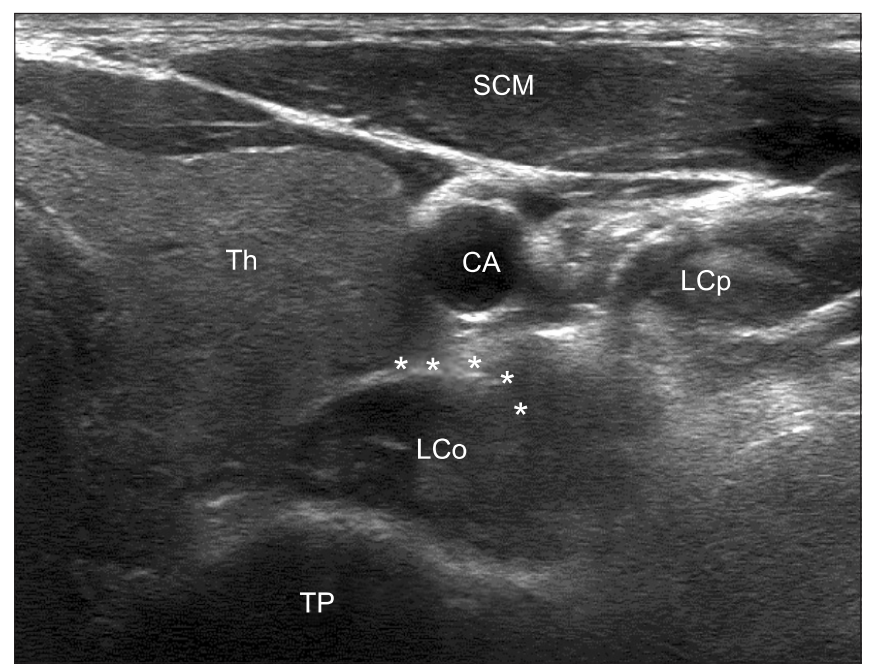

Fig. 1. Ultrasonography imaging of the left stellate ganglion \& prevertebral fascia (asterisks). Needle was advanced with real-time ultrasonography so that the needle tip will lie anterior to the longus coli. Th: Thyroid, LCo: Longus coli muscle, LCp: Longus capitus muscle, CA: Carotid artery, SCM: Sternocleidomastoid muscle, TP: Transverse process. which 0 represents 'no pain' and 10 represents 'the highest pain'. Determination of the volume of the hands (hand volume) was assessed by a hand volumeter. The hand volumeter set $(8 \times 13 \times 28 \mathrm{~cm})$ used was made of translucent material (acrylic), "Hand Volumeter" (Volumeter Unlimited, Phoenix, USA) and filled with room temperature water.

The volunteer was positioned beside the instrument during the procedure and the hand also positioned vertically, to avoid contact with the sides of the equipment. The hand was immersed slowly into the volumeter until it was immersed into water $2 \mathrm{~cm}$ above wrist crease. Additionally, the severity score for CRPS that was introduced by Harden in 2010 was evaluated as secondary outcome measure (Appendix 1). ${ }^{16}$ All these parameters were checked before procedure, after 2 weeks and 4 weeks following the SGB.

\section{Statistical analysis}

Statistical analysis was performed using Statistical Package for Social Sciences 11.0 version SPSS V11.0K (SPSS Inc., Chicago, USA). The Wilcoxon signed-rank test was used to compare VAS values and difference of hand volume before, 2 weeks and 4 weeks after the blockade for all patients and for each group. The Mann-Whitney U test was used for group comparisons on the mean differences of VAS values, hand volume and score of CRPS clinical severity. Null hypotheses of no difference were rejected if p-values were less than 0.05 .

\section{RESULTS}

Patient demographics are summarized in Table 1. There were no significant differences in characteristics included hemi-side, bone scan findings, and CRPS stage between the two groups. Within each group, VAS values were significantly decreased after two weeks and after four weeks ( $p$-value $<0.05$ ) (Table 2). Between each group, VAS reductions were $2.61 \pm 1.09,1.88 \pm 0.62$ at 2 weeks and $3.86 \pm 1.03,2.97 \pm 0.62$ at 4 weeks, respectively. The USguided SGB group showed more significant improvement in mean change of VAS compared with the blind SGB group (p-value<0.05) (Table 3 ).

SGB significantly improved in dealing the swelling of hand in both treatment groups at 2 weeks and 4 weeks, following SGB compared to pretreatment ( $p$-value $<0.05$ ) (Table 3). Between each group, differences of hand vol- 
Table 2. Changes of Visual Analogue Scale

\begin{tabular}{lccr}
\hline & Before injection & 2 weeks after injection & 4 weeks after injection \\
\hline Blind group & $5.09 \pm 1.13$ & $3.21 \pm 1.44$ & $2.12 \pm 1.06^{*}$ \\
US-guided group & $5.24 \pm 1.61$ & $2.63 \pm 1.64^{*}$ & $1.38 \pm 0.98^{*}$ \\
\hline
\end{tabular}

Values are mean \pm standard deviation

US: Ultrasonography

${ }^{*} \mathrm{p}<0.05$

Table 3. Intergroup Comparison of the Efficacy by Stellate Ganglion Block

\begin{tabular}{lcccccc} 
& \multicolumn{2}{c}{ 2 weeks after injection } & & \multicolumn{2}{c}{ 4 weeks after injection } \\
\cline { 2 - 3 } \cline { 5 - 6 } & Blind group & US-guided group & & Blind group & US-guided group \\
\hline$\Delta$ VAS & $1.88 \pm 0.62$ & $2.61 \pm 1.09 *$ & & $2.97 \pm 0.62$ & $3.86 \pm 1.03^{*}$ \\
$\Delta$ Volume of Hand $(\mathrm{ml})$ & $61.21 \pm 36.43$ & $67.38 \pm 27.73$ & & $86.67 \pm 33.94$ & $95.65 \pm 25.83$ \\
$\Delta$ Score of CSC & $1.91 \pm 1.40$ & $2.33 \pm 1.22$ & & $3.23 \pm 1.30$ & $3.69 \pm 1.63$ \\
\hline
\end{tabular}

Values are mean \pm standard deviation

VAS: Visual analogue scale, US: Ultrasonography, CSC: Clinical score of CRPS, $\Delta$ : Difference compared with baseline data

${ }^{*} \mathrm{p}<0.05$

ume were $61.2 \pm 36.4,67.4 \pm 27.7$ at 2 weeks and $86.7 \pm 33.9$, $95.7 \pm 25.8$ at 4 weeks. There were no significant differences between the two groups.

Additionally, score of CRPS clinical severity between the two groups didn't significantly decrease ( $\mathrm{p}$-value $>0.05$ ) (Table 3).

In US-guided group, no adverse effects to the procedure itself were documented during the procedure or followup period, but 2 patients from the blind group experienced a formation of hematoma on injected site. There were no long term sequelae.

\section{DISCUSSION}

The primary goal of this study was to evaluate the effects of US-guided SGB treatment on CRPS following stroke. Treatment produced a statistically significant difference in pain reduction and improvement of edema of upper extremity in both groups. However, US guided SGB was better in pain relief but has no advantages in reduction of hand swelling comparing with blind SGB.

The number of studies of SGB in the literature is limited. Cepeda published review in 2002, they concluded that there are questions as to the efficacy of local anesthetic sympathetic blockade in treating CRPS because its efficacy is based mainly on case series. ${ }^{9}$ Many imaging devices have been studied for enhancing the accuracy of SGB to prevent serious side effects. Among these, computerized tomography and MRI are considered to be time-consuming and expensive, and above of all, are impractical for most practitioners. ${ }^{17,18}$ Fluoroscopy has gained popularity for its convenience and the relatively familiar real-time imaging, but fluoroscopy still cannot prevent improper injections into other important anatomical structures, such as the esophagus, which may be accidentally punctured, leading to infection of the neck. ${ }^{19}$ Ultrasonography imaging can easily identify soft tissue around the sympathetic ganglion and this study showed that only two patients in blind group experienced shortterm complications on the injected site.

This study showed improvement of hand swelling in both groups but no advantages in reduction of hand swelling comparing with blind SGB. It may be that the etiology of post-stroke hand swelling is obscure. Vasomotor dysfunction, part of the unilateral disturbance of the autonomic nervous system that may follow stroke was mentioned as a causative factor. ${ }^{20}$ However, other possible explanations for post-stroke hand swelling are impaired lymphatic or venous drainage due to loss of muscle tone and of muscle pump activity. The possibility of increased capillary permeability in paretic limbs as the cause of post-stroke hand swelling was also raised. ${ }^{21}$ It follows that the role of sympathetic vasomotor dysfunction in post-stroke hand swelling formation is unclear. 
Therefore, reduction of hand swelling might be affected by various factors as well as SGB in our study.

We report that US-guided SGB successfully relieved pain in patients with CRPS following stroke, as indicated by a significant reduction in VAS values for all patients following the block. A relatively small sample and a wide range of conditions causing upper extremities pain following stroke limited in this study. The other weaknesses of this study were the lack of long-term results of the blockades and the lack of analysis based on CRPS stage. Further additional studies on these limitations will be required to identify the SGB efficacy which is related to the degree of symptoms. Despite these limitations, this study is a preliminary study which reveals that the efficacy of US-guided SGB is superior to blind SGB in post-stroke CRPS. US-guided sympathetic block treatment may be particularly helpful in cases when pain limits a patient's participation in stroke rehabilitation.

\section{CONCLUSION}

US-guided stellate ganglion block may improve the efficacy and safety of the procedure by direct visualization of the related anatomical structures and accordingly the risk of thyroid gland and vessels, vertebral artery, or esophagus injury may be minimized. Also ultrasound guidance will allow direct monitoring of the spread of the local anesthetics.

We concluded that treating post-stroke CRPS with USguided SGB successfully decreased VAS values compared to the classical blind approach technique. Additionally, compared with blind SGB, US-guided SGB can reduce the volume of anesthetic required to achieve therapeutic effect compared with blind SGB.

\section{REFERENCES}

1. Drummond PD, Finch PM, Skipworth S, Blockey P. Pain increases during sympathetic arousal in patients with complex regional pain syndrome. Neurology 2001; 57: 1296-1303

2. Chung OY, Bruehl SP. Complex regional pain syndrome. Curr Treat Options Neurol 2003; 5: 499-511

3. Zhang JM, Li H, Munir MA. Decreasing sympathetic sprouting in pathologic sensory ganglia: a new mechanism for treating neuropathic pain using lidocaine.
Pain 2004; 109: 143-149

4. Chung KS, Lee BH, Yoon YW, Chung JM. Sympathetic sprouting in the dorsal root ganglia of the injured peripheral nerve in a rat neuropathic pain model. J Comp Neurol 1996; 376: 241-252

5. McLachlan EM, Janig W, Devor M, Michaelis M. Peripheral nerve injury triggers noradrenergic sprouting within dorsal root ganglia. Nature 1993; 363: 543-546

6. Kumar V, Kalita J, Gujral RB, Sharma VP, Misra UK. A study of bone densitometry in patient with complex regional pain syndrome after stroke. Postgrad Med J 2001; 77: 519-522

7. Braus DF, Krauss JK, Strobel J. The shoulder hand syndrome after stroke: a prospective clinical trial. Ann Neurol 1994; 36: 728-733

8. Gellman H, Keenan MA, Stone L, Hardy SE, Waters RL, Stewart C. Reflex sympathetic dystrophy in brain injured patients. Pain 1992; 51: 307-311

9. Cepeda MS, Lau J, Carr DB. Defining the therapeutic role of local anesthetic sympathetic blockade in complex regional pain syndrome: a narrative and systematic review. Clin J Pain 2002; 18: 216-233

10. Higa K, Hirata K, Hirota K, Nitahara K, Shono S. Retropharyngeal hematoma after stellate ganglion block: analysis of 27 patients reported in the literature. Anesthesiology 2006; 105: 1238-1245

11. Mahli A, Coskun D, Akcali DT. Aetiology of convulsions due to stellate ganglion block. Eur J Anaesthesiol 2002; 19: 376-380

12. Kapral S, Krafft P, Gosch M, Fleischmann D, Weinstabl C. Ultrasound imaging for stellate ganglion block: direct visualization of puncture site and local anesthetic spread. A pilot study. Reg Anesth 1995; 20: 323-328

13. Narouze S, Vydyanathan A, Patel N. Ultrasoundguided stellate ganglion block successfully prevented esophageal puncture. Pain Physician 2007; 10: 747752

14. Harden RN, Bruehl S, Perez RS, Birklein F, Marinus J, Maihofner C, Lubenow T, Buvanendran A, Mackey S, Graciosa J, et al. Validation of proposed diagnostic criteria for complex regional pain syndrome. Pain 2010; 150: $268-274$

15. Elias M. Cervical sympathetic and stellate ganglion blocks. Pain Physician 2000; 3: 294-304

16. Harden RN, Bruehl S, Perez RS, Birklein F, Marinus J, Maihofner C, Lubenow T, Buvanendran A, Mackey S, 
Graciosa J, et al. Development of a severity score for CRPS. Pain 2010; 151: 870-876

17. Erickson SJ, Hogan QH. CT-guided injection of the stellate ganglion: description of technique and efficacy of sympathetic blockade. Radiology 1993; 188: 707 709

18. Hogan Q, Erickson SJ. Magnetic resonance imaging of the stellate ganglion: normal appearance. AJR Am J Roentgenol 1992; 158: 655-659

19. Siegenthaler A, Mlekusch S, Schliessbach J, Curatolo M, Eichenberger U. Ultrasound imaging to estimate risk of esophageal and vascular puncture after conventional stellate ganglion block. Reg Anesth Pain Med 2012; 37: 224-227

20. Redisch W, Tangco FT, Wertheimer L, Lewis AJ, Steele JM. Vasomotor responses in the extremities of subjects with various neurologic lesions. I. Reflex responses to warming. Circulation 1957; 15: 518-524

21. Exton-Smith AN, Crockett DJ. Nature of oedema in paralyzed limbs of hemiplegic patients. Br Med J 1957; 30: $1280-1283$ 
Appendix 1. Clinical Score of CRPS

\begin{tabular}{|c|c|c|c|}
\hline \multicolumn{2}{|c|}{ Self-reported symptoms } & \multicolumn{2}{|c|}{ Signs observed on examination } \\
\hline Allodynia, hyperpathia & 1 & Hyperpathia to pinprick & 1 \\
\hline & 0 & & 0 \\
\hline \multirow[t]{2}{*}{ Temperature asymmetry } & 1 & Allodynia & 1 \\
\hline & 0 & & 0 \\
\hline \multirow[t]{2}{*}{ Skin color asymmetry } & 1 & Temperature asymmetry by palpation & 1 \\
\hline & 0 & & 0 \\
\hline \multirow[t]{2}{*}{ Sweating asymmetry } & 1 & Skin color asymmetry & 1 \\
\hline & 0 & & 0 \\
\hline \multirow[t]{2}{*}{ Asymmetric edema } & 1 & Sweating asymmetry & 1 \\
\hline & 0 & & 0 \\
\hline \multirow[t]{2}{*}{ Trophic changes } & 1 & Asymmetric edema & 1 \\
\hline & 0 & & 0 \\
\hline \multirow[t]{2}{*}{ Motor changes } & 1 & Trophic changes & 1 \\
\hline & 0 & & 0 \\
\hline \multirow[t]{4}{*}{ Decreased active range of motion } & 1 & Motor changes & 1 \\
\hline & 0 & & 0 \\
\hline & & Decreased active range of motion & 1 \\
\hline & & & 0 \\
\hline
\end{tabular}

$1=$ presence $0=$ absence

Total: 17 points 